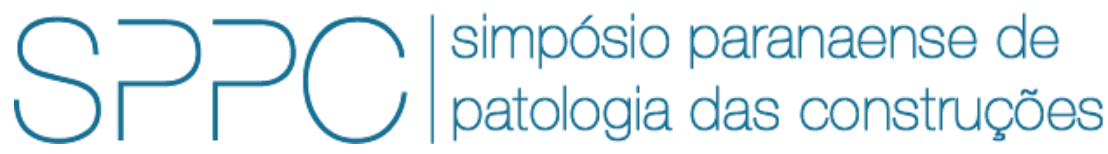

ISSN 2526-7248 artigo 2SPPC1002, pp. 14-23, 2017

\title{
Efeito da escória de alto forno na resistividade elétrica superficial do concreto
}

\author{
GANS, P. S. ${ }^{1}$; MEDEIROS-JUNIOR, R. A. ${ }^{2}$; LIMA, M. G. ${ }^{3}$ \\ ${ }_{1}^{1}$ Mestranda Eng. Civil, UFPR, patriciagans@hotmail.com \\ 2 Prof. Dr. Eng. Civil, UFPR, ronaldodemedeirosjr@yahoo.com.br \\ ${ }^{3}$ Profa. Dra. Eng. Civil, ITA, magdlima@gmail.com
}

\begin{abstract}
Resumo: Ao pensar em projetar estruturas de concreto a partir do conceito de durabilidade, a resistividade elétrica torna-se um parâmetro interessante para controle de vida útil, principalmente devido à praticidade de execução do ensaio, caracterizado como não destrutivo. A resistividade é definida como a habilidade do material em transportar cargas elétricas no seu interior, ou seja, no caso do concreto, de difundir os íons através da solução aquosa presente em seus poros. Relacionada à permeabilidade, a resistividade é um indicativo da resistência do concreto à penetração de agentes agressivos, a qual, consequentemente, afeta a velocidade de corrosão das armaduras do concreto. $O$ objetivo deste artigo é comparar a resistividade elétrica superficial (RES) de concretos com dois diferentes tipos de cimento: um com adição de escória de alto forno durante a composição (CP III RS) e outro sem esse tipo de adição (CP V). A RES foi medida pelo método dos quatro pontos. Foi utilizado o mesmo traço 1:1,4:2,1 (cimento: areia: brita) e a mesma relação água/cimento de 0,50 para as duas amostras de concreto investigadas. Os resultados foram analisados nas idades de 28, 60, 90, 120, 150 e 180 dias. Para os concretos com ambos os tipos de cimento foi identificado um aumento da RES com o tempo de ensaio. Porém, no cimento com adição de escória, o fator idade do concreto parâmetro que mede o crescimento da resistividade no tempo - elevou-se $45 \%$ em relação ao fator idade das amostras de CP V. A RES foi muito superior para o concreto com cimento de escória de alto forno em todas as idades, atingindo valor 9,7 vezes maior que amostra de concreto com cimento $\mathrm{CP}$ $\checkmark$ aos 180 dias.
\end{abstract}

Palavras-chave: concreto, resistividade elétrica superficial, escória de alto forno.

Abstract: The electrical resistivity is an interesting parameter of service life when thinking of durability of concrete structures, mainly due to the practicality of a non-destructive test method. In the case of concrete, it is defined as the material's ability to transport electric charges by diffusing the ions through the aqueous solution present in its pores. The electrical resistivity indicates the resistance of concrete to the penetration of aggressive agents that may cause corrosion of reinforcement. There are many factors that act directly on this parameter as the microstructure of concrete, the chemical composition of cement, the amount of water used in the mix and the proportion between materials. The aim of this study was to evaluate the change in surface electrical resistivity (SER) of concretes produced with two different types of cement: the blast-furnace slag cement (CP III RS) and a cement without this addition (CP V). It was used the same proportion between materials and the same water/cement ratio for the samples. The SER was measured using the Werner Method (four-point method) for 28, 60, 90, 120, 150 and 180 days. The results indicated that the SER increased with time for both types of cement, although the age factor - parameter that measures the development in resistivity over time - of the concrete with CP III RS was $45 \%$ higher. This concrete had better results in every age and it was almost tenfold for 180 days when comparing with samples produced with CP V.

Keywords: concrete, surface electrical resistivity, blast furnace slag. 
GANS, P. S.; MEDEIROS-JUNIOR, R. A.; LIMA, M. G. EFEITO DA ESCÓRIA DE ALTO FORNO NA RESISTIVIDADE ELÉTRICA SUPERFICIAL DO CONCRETO. $2^{\circ}$ Simpósio Paranaense de Patologia das Construções (20 SPPC), artigo 2SPPC1002, pp. 14-23,

\section{Introdução}

As normas em vigor de dimensionamento de estruturas têm se provado incapazes de garantir durabilidade suficiente para estruturas em ambientes agressivos. Por isso, é de interesse crescente na Construção Civil projetar baseado na durabilidade e desenvolver métodos de predição de vida útil [1].

Pode-se definir vida útil como o período de uso da estrutura no qual um desempenho minimamente aceitável é mantido, isto é, em que a resistência é maior que a solicitação e as características do concreto, segurança e estabilidade são mantidas [2]. A degradação ocorre quando há perda do desempenho da estrutura.

Um dos processos de degradação que mais comprometem a vida útil de estruturas é a corrosão das armaduras. Avaliações técnicas feitas nos países ibero-americanos classificaram as principais causas de deterioração das estruturas de concreto, e os danos provenientes da corrosão de armaduras estão entre as três primeiras causas [3].

A corrosão da armadura é assunto bastante discutido na literatura. Esse processo eletroquímico causa alterações prejudiciais indesejadas, como a redução da área de aço, redução da tensão de escoamento do aço, fissuração do concreto de cobrimento causada pelos produtos expansivos das reações químicas e compromete a aderência entre armadura e concreto, tornando a estrutura inadequada para uso [4].

Segundo Helene [5], a resistividade elétrica caracteriza a dificuldade dos íons se movimentarem no concreto, ou seja, é uma propriedade dos materiais em geral. Para o concreto, a resistividade controla o fluxo de íons difundidos em seu interior através da solução aquosa dos seus poros, ou seja, é o inverso da condutibilidade.

A difusão de íons cloreto nos poros do concreto é reconhecidamente um dos fatores de maior importância no desencadeamento do processo corrosivo, afetando, principalmente, estruturas em ambiente marinho [6]. Assim, relaciona-se com a importância da resistividade elétrica no estudo da durabilidade do concreto.

O fenômeno eletroquímico que gera o processo de corrosão das armaduras do concreto pode ser controlado, principalmente, pela resistividade elétrica e pela disponibilidade de oxigênio às barras da armadura. Portanto, a resistividade é um indicador de durabilidade. Quanto maior o seu valor, menor é a aptidão de desencadear o processo corrosivo [7].

A resistividade elétrica é sensível à microestrutura da matriz de concreto e as características da solução presente nos poros. Portanto, mudanças na proporção de materiais da mistura, na relação água/cimento e na utilização de aditivos e adições afetam diretamente essa propriedade [8].

Ao misturar adições minerais ao clínquer, a fim de produzir cimentos diferenciados, ocorrem mudanças na matriz do concreto. Essas mudanças são decorrentes da reação química entre os componentes do cimento e a água, e podem impactar nas propriedades elétricas do concreto. 
GANS, P. S.; MEDEIROS-JUNIOR, R. A.; LIMA, M. G. EFEITO DA ESCÓRIA DE ALTO FORNO NA RESISTIVIDADE ELÉTRICA SUPERFICIAL DO CONCRETO. $2^{\circ}$ Simpósio Paranaense de Patologia das Construções (20 SPPC), artigo 2SPPC1002, pp. 14-23,

A utilização das adições minerais no concreto decorre de vários fatores, desde econômicos até ecológicos, e pode ser justificada pelas melhorias que causam em certas características do concreto, como a durabilidade.

O objetivo deste trabalho é avaliar o efeito da adição de escória de alto forno presente no cimento, classificada como uma adição mineral cimentante, na resistividade elétrica do concreto.

\section{Programa Experimental}

Para que fosse possível comparar o efeito da adição de escória de alto forno na resistividade elétrica superficial do concreto, foram selecionados dois tipos de cimento para produção dos corpos de prova: cimento Portland de alto forno, classe 40, resistente ao sulfato (CP III 40 RS), e cimento Portland de alta resistência inicial (CP V ARI). O CP III 40 RS possui adição de até $70 \%$ de escória de alto forno em sua composição, já o CP V é basicamente formado por clínquer e sulfatos de cálcio.

As caraterísticas químicas dos dois cimentos, segundo os limites das normas ABNT NBR 5735 [9], ABNT NBR 5737 [10] e ABNT NBR 5733 [11] estão apresentadas na Tabela 1.

Tabela 1: Propriedades químicas: Limites de normas brasileiras

\begin{tabular}{ccc}
\hline $\begin{array}{c}\text { Propriedades químicas (unidade: } \\
\text { \% em volume) }\end{array}$ & CP III RS & CP V \\
\hline Norma ABNT & NBR 5735 e NBR 5737 & NBR 5733 \\
\hline Clínquer + sulfatos de cálcio & $40-25$ & $100-95$ \\
Escória granulada de alto forno & $60-70$ & - \\
Material pozolânico & - & - \\
Material carbonático & $0-5$ & $0-5$ \\
Óxido de magnésio (MgO) & - & 6,5 \\
Trióxido de enxofre (SO3) & 4,0 & - \\
Perda ao fogo & 4,5 & 4,5 \\
Resíduos Insolúveis & 1,5 & 1,0 \\
Anidrido Carbônico (CO 2 ) & 3,0 & 3,0 \\
\hline
\end{tabular}

Como agregado miúdo, foi utilizada areia natural proveniente de rio, disponível comercialmente na cidade de São José dos Campos, com dimensão máxima característica de 2,36mm e módulo de finura 2,6. O agregado graúdo utilizado foi brita comercial com dimensão máxima de $9,5 \mathrm{~mm}$ e módulo de finura 6,5 .

Foi fixado o traço 1:1,4:2,1 (cimento: areia: brita), em materiais secos, e a relação água/cimento de 0,50 para fabricação de todos os corpos de prova. Portanto, a relação entre cimento e agregados usada foi 1:3,5, com consumo de cimento de $474 \mathrm{~kg} / \mathrm{m}^{3}$.

Baseado na experiência de ensaios discutida por Gowers e Millard [12] e Lencioni


moldados 4 corpos de prova para cada tipo de concreto. Para tal, foram utilizadas 
formas de madeira, adensados com vibrador de imersão em 3 camadas, e desmoldados após 48 horas da moldagem.

Os corpos de prova permaneceram com a superfície coberta e umedecida até o momento da desmoldagem, para evitar troca de água com a superfície. Após esse momento, ocorreu a cura saturada em câmara úmida (umidade relativa do ar maior que 95\%), durante um período de 28 dias. Então, foram colocados em ambiente de laboratório até as idades de realização dos ensaios.

O ensaio de resistividade elétrica superficial (RES) foi realizado aos 28, 60, 90, 120 , 150 e 180 dias pelo Método de Werner, ou método dos quatro pontos. Esse método consiste em utilizar um equipamento que possui quatro contatos espaçados a igual distância $(\mathrm{d}=0,05 \mathrm{~m})$. Ao ser posicionado sobre a superfície do concreto, uma corrente elétrica é aplicada entre os dois contatos externos e a diferença de potencial resultante é medida pelos dois contatos internos.

O valor da RES é determinado pela Equação 1 [14]. Um esquema desse procedimento é ilustrado na Figura 1.

$$
\rho=R \cdot \frac{A}{L}
$$

Em que: $\rho=$ resistividade elétrica $(\Omega . m) ; R=$ resistência elétrica $(\Omega)$, determinada através da aplicação de uma diferença de potencial $(V$, em volts) entre dois pontos e medindo a corrente ( $\mathrm{I}$, em ampere) resultante; $\mathrm{A}=$ área da seção reta do material condutor $\left(\mathrm{m}^{2}\right)$; e $\mathrm{L}=$ distância entre os eletrodos $(\mathrm{m})$.

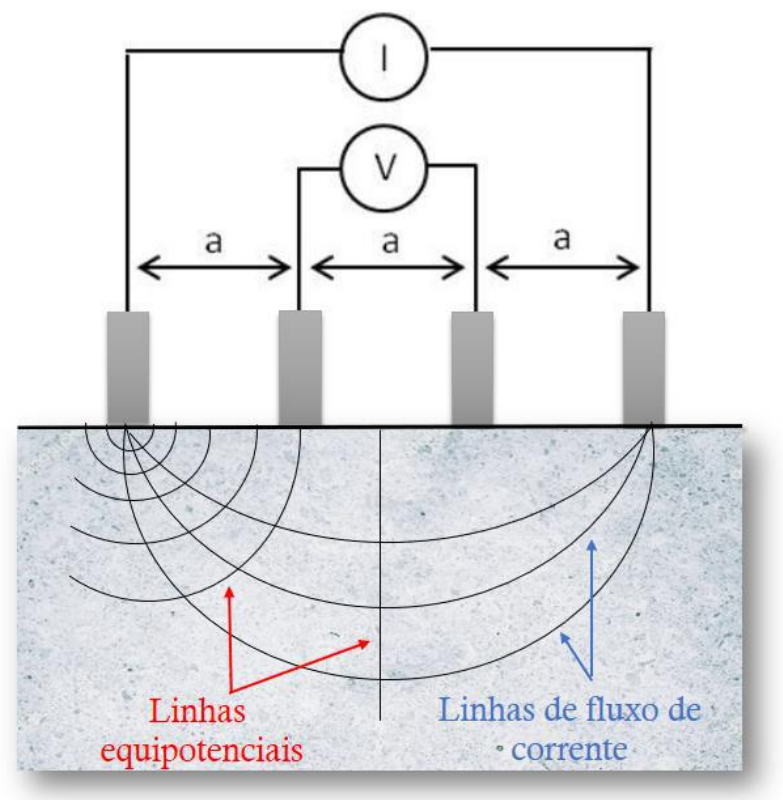

Figura 1: Esquema da Técnica de Werner para medida da RES do concreto (Gowers e Millard [12] traduzido por Medeiros-Junior [6])

Com os dados de RES obtidos durante o monitoramento contínuo dos corpos de prova, o fator idade ( $q$ ) foi obtido pela Equação 2. Esse fator mede a evolução da RES ao longo do tempo através de um ajuste de potência, em que q é o expoente [15]. 


$$
\rho_{2}=\rho_{0}\left(\frac{t_{2}}{t_{0}}\right)^{q}
$$

Em que: $\rho_{2}=$ resistividade elétrica $(\Omega . \mathrm{cm})$ no tempo t2 (anos); $\rho_{0}=$ resistividade elétrica $(\Omega . c m)$ na idade (to, em anos) da primeira medição (28 dias de idade, após cura saturada do concreto); e $\mathrm{q}=$ fator idade.

\section{Resultados e Discussões}

Nas Figuras 2 a 3 estão apresentados os resultados do ensaio de RES para as amostras contendo o cimento CP V e CP III RS, respectivamente, durante o período de monitoramento de 180 dias. Os resultados representam a média dos valores de RES de quatro corpos de prova para cada tipo de concreto investigado.

Durante o ensaio de RES, foi verificado que a dimensão dos corpos de prova utilizados é grande o suficiente para ser considerada como meio semi-infinito. Isso significa que as linhas de correntes geradas pelo equipamento no interior do concreto se fecham e, assim, não alteram os valores de RES obtidos.

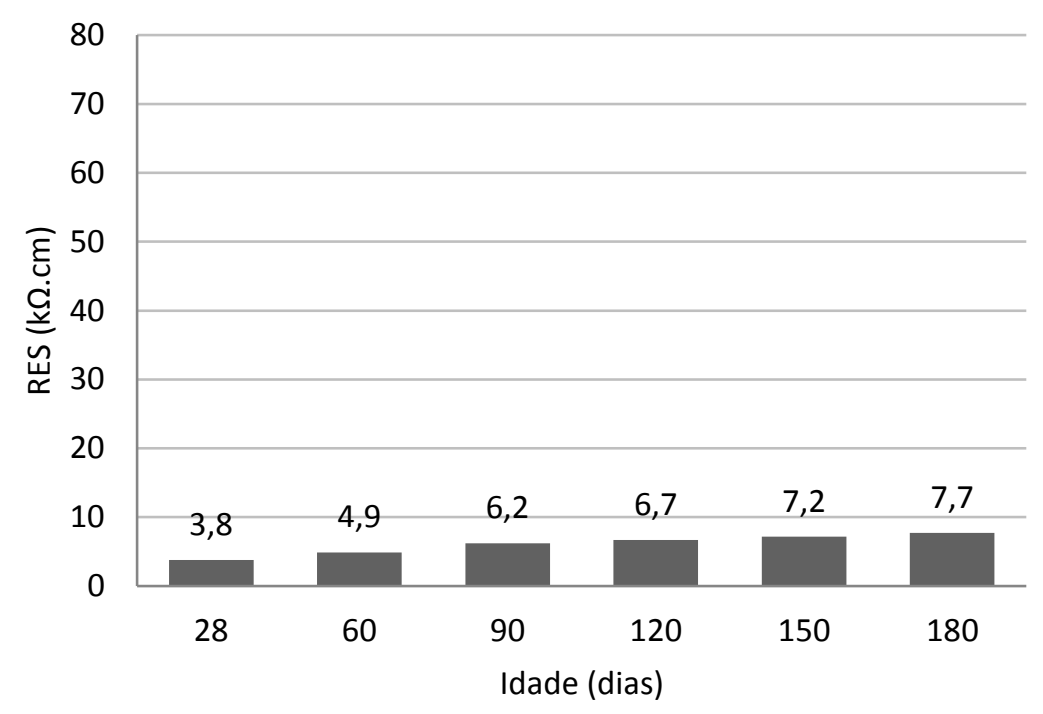

Figura 2: Resistividade elétrica superficial (RES) ao longo do tempo, para os concretos de cimento CP V 


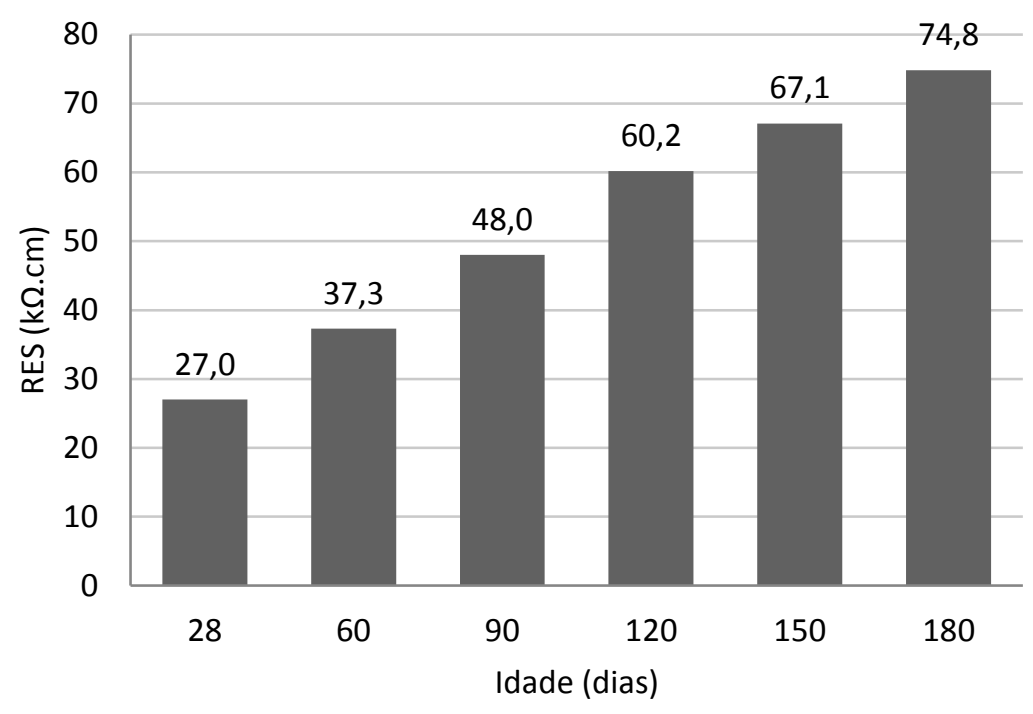

Figura 3: Resistividade elétrica superficial (RES) ao longo do tempo, para os concretos de cimento CP III RS

Observou-se que a RES do concreto com cimento CP III RS foi muito superior aos valores obtidos para o concreto com cimento CP V. Aos 28 dias, a RES desse concreto foi 7,1 vezes maior que o resultado das amostras de concreto com cimento CP V. Já aos 180 dias, a média dos resultados do ensaio atingiu valor 9,7 vezes superior.

Esse resultado pode ser atribuído à alta quantidade de adição de escória no cimento CP III RS (cerca de 60 a 70\%, conforme Tabela 1). A presença de escória no cimento aumenta a densidade do concreto, assim como a resistência da matriz e a sinuosidade capilar, além de reduzir a concentração iônica nos poros [16,17]. Portanto, ao refinar os poros da microestrutura do concreto, é diminuída a condutividade das amostras e, consequentemente, aumenta-se a RES.

Resultados em concordância já foram obtidos na bibliografia. Gesoğlu e Özbay [18] compararam amostras de concreto com cimento com adição de escória e sem adição e encontrou valores mais elevados de RES no primeiro grupo. Lübeck et al. [19] fez amostras de concreto com $50 \%$ de substituição do cimento Portland comum por escória de alto forno e observou aumento de 2,5 a 4,8 vezes nos valores de RES aos 7 dias, quando comparadas ao concreto sem nenhuma adição.

De acordo com as Figuras 3 e 4, houve um crescimento da RES com o tempo para ambos os concretos. Isso ocorre porque, nas primeiras horas, o concreto apresenta maior condutividade elétrica. Com a mistura dos materiais, há a formação de uma pasta de cimento e água, e a água, por conter íons dissolvidos, funciona como condutor no interior da massa de concreto.

A RES aumenta com o tempo devido à evolução das reações de hidratação e endurecimento do concreto. Esse comportamento também foi identificado por Baweja et al. [20], CCAA [21], Andrade e D'Andréa [22], Presuel-Moreno et al. [23] e Medeiros-Junior e Lima [15]. 
GANS, P. S.; MEDEIROS-JUNIOR, R. A.; LIMA, M. G. EFEITO DA ESCÓRIA DE ALTO FORNO NA RESISTIVIDADE ELÉTRICA SUPERFICIAL DO CONCRETO. $2^{\circ}$ Simpósio Paranaense de Patologia das Construções (20 SPPC), artigo 2SPPC1002, pp. 14-23,

A variação da RES com o tempo, para ambos os tipos de cimentos, é representada na Figura 4, em que to é a idade da primeira medida de ensaio, ou seja, 28 dias. Um ajuste de potência foi feito para as amostras de cada cimento, a fim de encontrar o fator idade $q$.

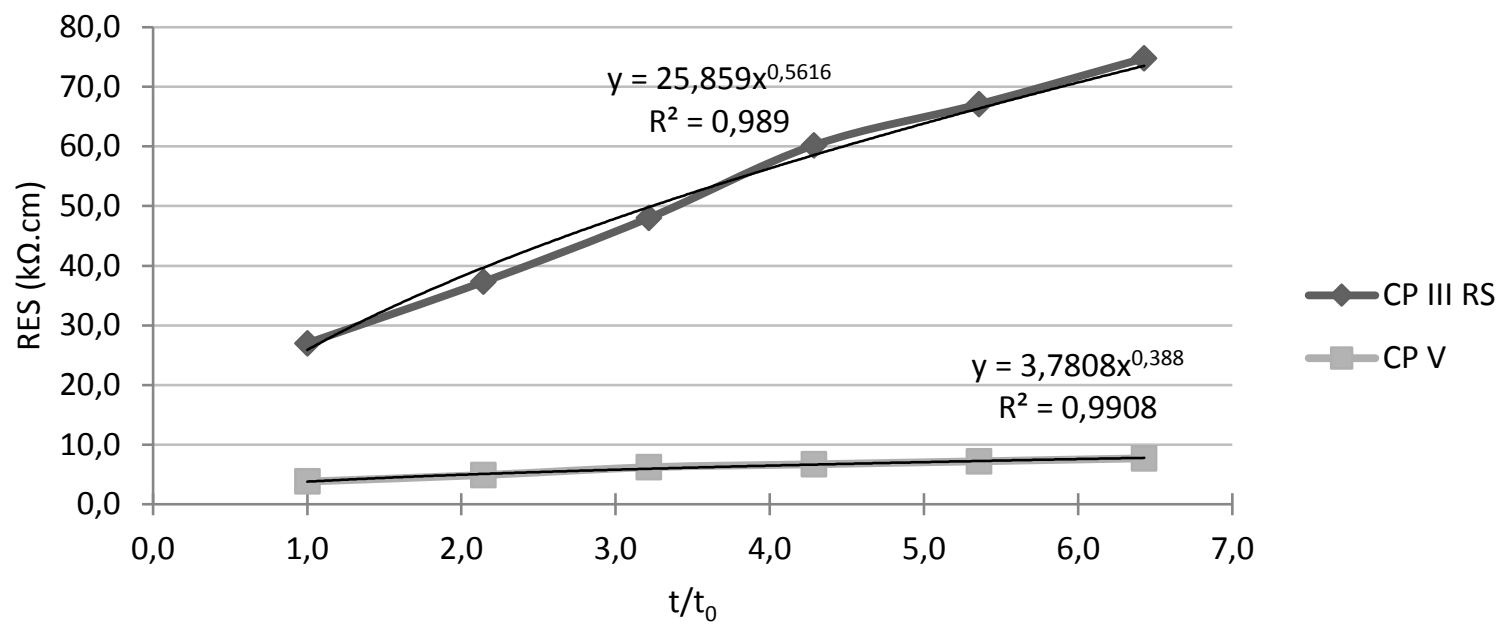

Figura 4: Evolução da RES no tempo

De acordo com os resultados, o cimento com adição de escória de alto forno, além de apresentar maiores valores absolutos de RES, também apresentou maior evolução com o tempo. Ao comparar a RES aos 28 e 180 dias, houve um crescimento de $177 \%$ nas amostras de cimento CP III RS, enquanto para as amostras de CP V, o crescimento foi de $102 \%$.

Através da aplicação da Equação 2 nos dados da Figura 5, expresso pelo ajuste de potência, foi encontrado um fator idade igual a 0,5616 para o concreto com cimento CP III RS, valor $45 \%$ maior ao fator idade do concreto com cimento CP V, igual a 0,388 .

Tal fenômeno pode ser explicado pela adição mineral reagir progressivamente com a água. Isto é, quando comparada ao clínquer puro, as reações de hidratação da escória são mais lentas, porém progressivas.

Ao observar o ajuste dos resultados da série contendo cimento CPV, nota-se que há uma estabilização mais rápida da RES, em função do menor acréscimo no tempo. Já para a série contendo CP III RS, não há sinal de estabilização no período de medição estabelecido. Esse estudo continua em andamento com o monitoramento da RES em um período mais longo, a fim de encontrar a tendência de estabilização para esse tipo de cimento.

\section{Conclusão}

As amostras de concreto com cimento CP III RS, o qual contém adição de escória de alto forno, apresentam maiores valores de RES para todas as idades. Na data mais avançada investigada nesse estudo (180 dias), a RES dessas amostras é 9,7 vezes maior que o valor das amostras de concreto com cimento CP V. 
GANS, P. S.; MEDEIROS-JUNIOR, R. A.; LIMA, M. G. EFEITO DA ESCÓRIA DE ALTO FORNO NA RESISTIVIDADE ELÉTRICA SUPERFICIAL DO CONCRETO. $2^{\circ}$ Simpósio Paranaense de Patologia das Construções (2० SPPC), artigo 2SPPC1002, pp. 14-23,

A RES também aumenta com o tempo de ensaio, resultado da hidratação e endurecimento do concreto. Para as amostras de concreto com cimento CP III RS, a evolução com o tempo é maior, quando comparado às amostras de concreto com cimento CP V. O fator idade do concreto com adição mineral é $45 \%$ superior ao sem nenhuma adição.

Conclui-se que a adição de escória de alto forno na composição do cimento contribui significativamente para o aumento da resistividade elétrica superficial do concreto.

\section{Agradecimentos}

À Universidade Federal do Paraná (UFPR), em especial ao Programa de PósGraduação em Engenharia de Construção Civil (PPGECC), e ao Instituto Tecnológico de Aeronáutica (ITA).

\section{Referências}

[1] ANDRADE, C.; D'ANDRÉA, R. (2010) Electrical resistivity as microstructural parameter for the modeling of service life of reinforced concrete structures. In.: 2nd International Symposium on Service Life Design for Infrastructure. Delft, The Netherlands, p. 379- 388.

[2] NBR 6118 (2014) Projeto de estruturas de concreto - Procedimento. Associação Brasileira de Normas Técnicas - ABNT, Rio de Janeiro, Brasil.

[3] RINCÓN, O. T. (2006) Durability of concrete structures: DURACON, an Iberoamerican project. Preliminary results Building and Environment. v. 41, p. 952-962.

[4] PELLIZZER, G. P. (2015) Análise mecânica e probabilística da corrosão de armaduras de estruturas de concreto armado submetidas à penetração de cloretos. Dissertação (Mestrado) - Escola de Engenharia de São Carlos. São Carlos.

[5] HELENE, P. (1993) Contribuição ao Estudo da Corrosão em Armaduras de Concreto Armado. Tese (Livre Docência) - Escola Politécnica da Universidade de São Paulo. São Paulo.

[6] MEDEIROS-JUNIOR, R. A. (2014) Estudo da resistividade do concreto para proposta de modelagem de vida útil - Corrosão das armaduras devido à penetração de cloretos. Tese (Doutorado) - Instituto Tecnológico de Aeronáutica. São José dos Campos, São Paulo.

[7] LENCIONI, J. W.; DE LIMA, M. G. (2010) Principais fatores intervenientes nas medidas de resistividade elétrica superficial do concreto-Estado-da-Arte. In: VI Congresso Internacional sobre Patologia e Reabilitação de Estruturas. Córdoba, Argentina.

[8] POLDER, R. B. (2001) Test methods for on site measurement of resistivity of concrete-a RILEM TC-154 technical recommendation. Construction and building materials, v. 15, n. 2, p. 125-131. 
GANS, P. S.; MEDEIROS-JUNIOR, R. A.; LIMA, M. G. EFEITO DA ESCÓRIA DE ALTO FORNO NA RESISTIVIDADE ELÉTRICA SUPERFICIAL DO CONCRETO. $2^{\circ}$ Simpósio Paranaense de Patologia das Construções (20 SPPC), artigo 2SPPC1002, pp. 14-23,

[9] NBR 5735 (1991) Cimento Portland de alto-forno. Associação Brasileira de Normas Técnicas - ABNT, Rio de Janeiro, Brasil.

[10] NBR 5737 (1992) Cimentos Portland resistentes a sulfatos. Associação Brasileira de Normas Técnicas - ABNT, Rio de Janeiro, Brasil.

[11] NBR 5733 (1991) Cimento Portland de alta resistência inicial. Associação Brasileira de Normas Técnicas - ABNT, Rio de Janeiro, Brasil.

[12] GOWERS, K. R.; MILLARD, S. G. (1999) Measurement of concrete resistivity for assessment of corrosion severity of steel using wenner technique. $\mathrm{ACl}$ Materials Journal, v. 96-M66, p. 536-541.

[13] LENCIONI, J. W. (2011) Estudos sobre resistividade elétrica superficial em concreto: análise e quantificação de parâmetros intervenientes nos ensaios. Tese (doutorado) - Programa de Pós-Graduação em Engenharia de Infraestrutura Aeronáutica. Instituto Tecnológico de Aeronáutica - ITA. São José dos Campos, São Paulo.

[14] HALLIDAY, D.; RESNICK,R. (2009) Fundamentos da física. Eletromagnetismo. $8^{\mathrm{a}}$ ed. Rio de janeiro: LCT, v. 3.

[15] MEDEIROS-JUNIOR, R. A.; LIMA, M. G. (2016) Electrical resistivity of unsaturated concrete using different types of cement. Construction and Building Materials, v. 107, p. 11-16.

[16] BIJEN, J. (1996) Benefits of slag and fly ash. Construction and building materials, v. 10, n. 5, p. 309-314.

[17] MCCARTER, W. J.; STARRS, G.; CHRISP, T. M. (2000) Electrical conductivity, diffusion, and permeability of Portland cement-based mortars. Cement and Concrete Research, v. 30, n. 9, p. 1395-1400.

[18] GESOĞLU, M.; ÖZBAY, E. (2007) Effects of mineral admixtures on fresh and hardened properties of self-compacting concretes: binary, ternary and quaternary systems. Materials and Structures, v. 40, n. 9, p. 923-937.

[19] LÜBECK, A.; GASTALDINI, A. L. G.; BARIN, D. S.; SIQUEIRA, H. C. (2012) Compressive strength and electrical properties of concrete with white Portland cement and blast-furnace slag. Cement and Concrete Composites, v. 34, p. 392-399.

[20] BAWEJA, D.; ROPER, H.; SIRIVIVATNANON, V. (1997) Quantitative descriptions of steel corrosion in concrete using resistivity and anodic polarisation data. In.: Proceedings 4th CANMET/ACI International Conference on Durability of Concrete. V. M. Malhotra (ed.), SP 170-3, pp 41-63.

[21] CCAA - CEMENT CONCRETE \& AGGREGATES AUSTRALIA. (2009) Chloride Resistance of Concrete. Report.

[22] ANDRADE, C.; D’ANDRÉA, R. (2011) La resistividad eléctrica como parámetro de control del hormigón y de su durabilidad. Revista ALCONPAT, v. 1, n. 2, p. 93-101. 
GANS, P. S.; MEDEIROS-JUNIOR, R. A.; LIMA, M. G. EFEITO DA ESCÓRIA DE ALTO FORNO NA RESISTIVIDADE ELÉTRICA SUPERFICIAL DO CONCRETO. $2^{\circ}$ Simpósio Paranaense de Patologia das Construções (20 SPPC), artigo 2SPPC1002, pp. 14-23, 2017. DOI: $10.4322 / 2$ SPPC. 2017.002

[23] PRESUEL-MORENO, F.; WU, Y.-Y.; LIU, Y. (2013) Effect of curing regime on concrete resistivity and aging factor over time. Construction and Building Materials, v. 48, p. 874-882. 